\title{
Wilson disease diagnosed incidentally by targeted gene panel sequencing in a Korean boy with severe obesity
}

\author{
Minji Im? \\ Ari Song ${ }^{2}$, \\ Jiyeon $\mathrm{Kim}^{3}$, \\ Min-Sun Kim ${ }^{3}$ \\ Sae-Mi Lee, ${ }^{4,5}$, \\ Mi Jin $\mathrm{Kim}^{3}$, \\ Sung Yoon $\mathrm{Cho}^{3}$, \\ Dong-Kyu Jin ${ }^{3}$
}

'Department of Pediatrics, Myongji Hospital, Hanyang University Medical Center, Goyang, Korea

${ }^{2}$ Department of Pediatrics, Mediplex Sejong Hospital, Incheon, Korea

${ }^{3}$ Department of Pediatrics, Samsung Medical Center, Sungkyunkwan University School of Medicine, Seoul, Korea ${ }^{4}$ GC Genome, GCLabs, Yongin, Korea ${ }^{5}$ Department of Laboratory Medicine, Kangwon National University School of Medicine, Chuncheon, Korea

Received: 26 February, 2021

Revised: 26 May, 2021

Accepted: 22 June, 2021

Address for correspondence: Sung Yoon Cho

Department of Pediatrics, Samsung Medical Center, Sungkyunkwan University School of Medicine, 81 Irwon-ro, Gangnam-gu, Seoul 06351, Korea

Email: nadri1217@naver.com

https://orcid.org/0000-0003-2913059X
Wilson disease (WD) is a relatively common genetic hepatic disease in children and is characterized by excessive copper accumulation, predominantly in the liver and brain. It is an autosomal recessive disease caused by an ATP7B mutation that causes brain degeneration and is potentially fatal if diagnosed late or untreated. In the early phase of WD, its initial presentation may include mild hepatic involvement. WD may be overlooked as a cause of liver disease due to severe obesity but should not be excluded from differential diagnosis. We report a case of WD with severe obesity and fatty liver diagnosed in the early phase by targeted gene panel sequencing and review the endocrine problems associated with WD. Early suspicion of WD is important for good prognosis.

Keywords: Obesity, Nonalcoholic fatty liver disease, Hepatolenticular degeneration

\section{Highlights}

- We report a male aged 4 years 7 months with obese Wilson's disease (WD) accompanied by nonalcoholic fatty liver disease. We diagnosed him with WD incidentally based on targeted gene panel sequencing to screen for genes related to pathologic obesity. Early suspicion and diagnosis are important in this treatable and reversible disease.

\section{Introduction}

Wilson disease (WD, OMIM \#277900), or progressive hepatolenticular degeneration, is an autosomal recessive inherited disorder affecting copper metabolism whose incidence is estimated to be 1/30,000 in most populations. ${ }^{1)}$ In this disease, copper secretion from the liver into the bile fails, and copper is incorporated into ceruloplasmin. Copper primarily accumulates in the liver and brain tissue, so this disease usually manifests early as chronic liver disease and later as a neurological disorder. Hepatic manifestations can include any type of liver disease, from acute to chronic. WD is fatal if left untreated, but timely treatment can prevent severe liver disease and lifelong neurologic disabilities.

WD is usually suspected if the serum ceruloplasmin is low in patients with elevated liver enzyme levels, and its diagnosis relies on detection of Kayser-Fleischer rings, low ceruloplasmin, elevated urine and hepatic copper levels, signs of liver or neurologic disease, or associated histologic liver changes. Recent studies have reported on diagnosed WD cases with atypical symptoms through genetic testing to identify homozygous or complex heterozygous mutations in the $A T P 7 B$ gene.

Obesity is not a common feature in WD, is induced by decrease in energy expenditure, and has variable phenotypic severity. Common characteristics of obesity include insulin-resistance, hyperlipidemia, and nonalcoholic fatty liver disease (NAFLD). NAFLD is a spectrum of 
liver diseases strongly associated with obesity and is the most common chronic liver disease in children. It is also commonly observed in $\mathrm{WD}$, and it is the most frequently observed source of hepatic lesions. ${ }^{2)}$ Copper accumulation might be related to NAFLD pathogenesis. ${ }^{3)}$

Here we report a boy diagnosed with WD via genetic testing for severe obesity, along with a comprehensive literature review of the endocrine complications associated with WD.

\section{Case report}

The patient was born at 37 weeks gestational age through in vitro fertilization with a birth weight of $3,000 \mathrm{~g}$ ( $z$-score, -0.5 standard deviation score [SDS]). His body measurements, including birth height and head circumference, were within normal range. Meconium staining was not performed, and his vital signs were stable at birth. His APGAR score was 7 or higher. Laryngomalacia was observed until one month of age and improved naturally. There was no evidence of chorioamnionitis. Prenatal testing results, including amniocentesis, were normal. He was an only child and born to Korean non-consanguineous parents. His father had obesity, asthma, hypertension, and diabetes. Paternal height was $180 \mathrm{~cm}$ (1.2 SDS) and weight was $117 \mathrm{~kg}$ (4.2 SDS). Maternal height was $162 \mathrm{~cm}$ (0.1 SDS) and weight was $56 \mathrm{~kg}(-0.3 \mathrm{SDS})$. His grandparents had hypertension and diabetes. At 10 months old, he began toe walking, and his feet trembled when he was in a standing position due to spasticity of the lower limbs. He was diagnosed with cerebral palsy at the age of 13 months. At this point, height, weight, and body mass index (BMI) were $87.8 \mathrm{~cm}$ (2.9 SDS), 14.7
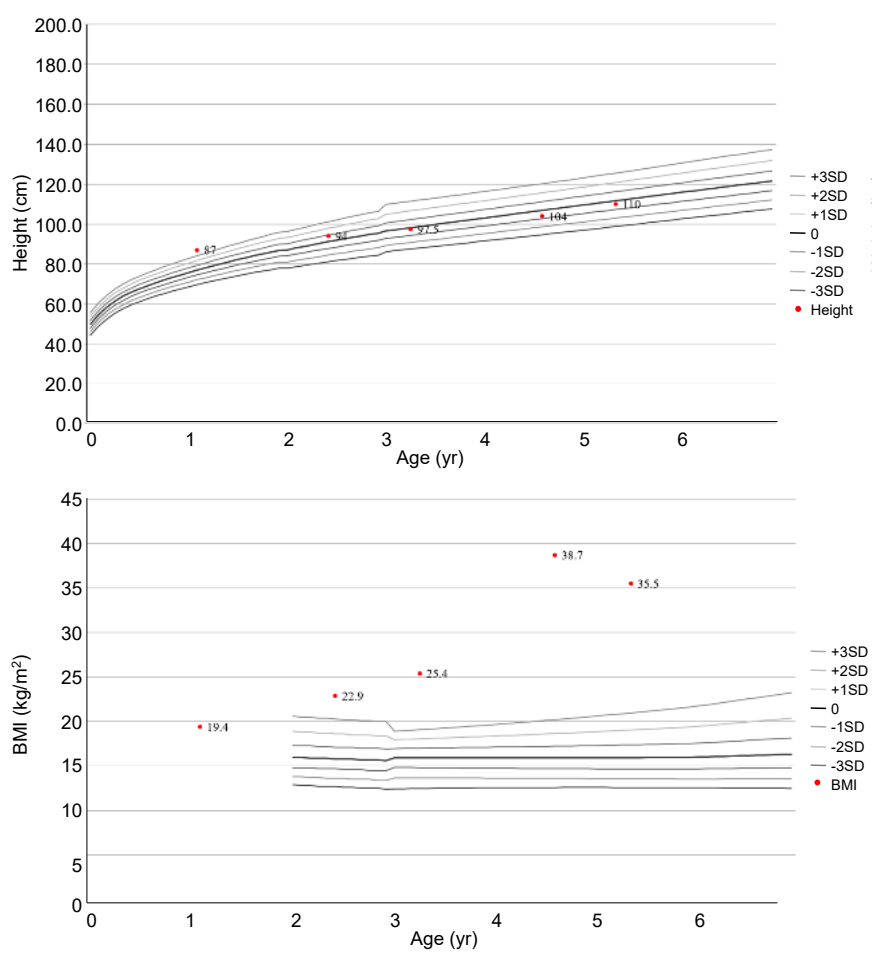

$\mathrm{kg}(3.5 \mathrm{SDS})$, and $18.8 \mathrm{~kg} / \mathrm{m}^{2}$, respectively. Brain magnetic resonance imaging showed ventriculomegaly with prominent septum cavum pellucidum, which indicated periventricular leukomalacia. He primarily consumed formula until the age of 24 months. His height, weight, and BMI were $94 \mathrm{~cm}$ (0.9 SDS), $20.2 \mathrm{~kg}$ (4.2 SDS), and $22.7 \mathrm{~kg} / \mathrm{m}^{2}$ (5.4 SDS) at 29 months old. He underwent rehabilitation treatment for cerebral palsy and nutritional counseling for obesity.

At the age of 4 years 7 months, the patient visited our department due to severe obesity. His height and weight were $104 \mathrm{~cm}(-0.5$ SDS $)$ and $41.9 \mathrm{~kg}$ (10.2 SDS), respectively, and his BMI was $38.7 \mathrm{~kg} / \mathrm{m}^{2}$ (17.5 SDS) (Fig. 1). He had small hands and feet and showed severe truncal obesity. His cognition and language development were that of an 8-10-month-old, and his motor development was that of a 10-12-month-old. Laboratory tests revealed elevated aspartate aminotransferase (AST, $98 \mathrm{U} / \mathrm{L}$; normal range, 0-40 U/L); elevated alanine aminotransferase (279 U/L; normal range, 0-41 U/L); elevated alkaline phosphatase (282 U/L; normal range, 40-129 U/L); normal total bilirubin $(0.2 \mathrm{mg} / \mathrm{dL})$; normal total cholesterol $(172 \mathrm{mg} / \mathrm{dL})$; elevated triglycerides $(181 \mathrm{mg} / \mathrm{dL}$; normal range, 0-149 mg/dL); normal high-density lipoprotein cholesterol (53 $\mathrm{mg} / \mathrm{dL}$ ), normal low-density lipoprotein cholesterol (99 mg/ $\mathrm{dL})$; normal glucose $(96 \mathrm{mg} / \mathrm{dL})$; elevated insulin $(27.0 \mu \mathrm{IU} /$ $\mathrm{mL}$; normal range 1.1-11.2 $\mu \mathrm{IU} / \mathrm{mL}$ ); and normal glycosylated hemoglobin (5.3\%). His parents were aware of his elevated liver enzymes when they visited our clinic, and he had previously been diagnosed with NAFLD. He did not present with jaundice. Abdominal ultrasound revealed severe fatty liver. His viral and autoimmune hepatitis work-up was normal.

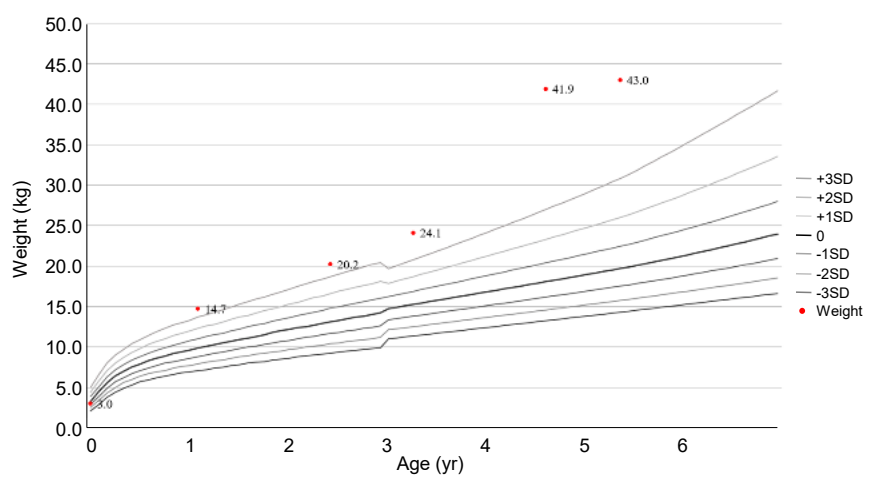

Fig. 1. The changes in patient growth curves and BMI-SDS. His weight remained above the 95th percentile for age from 13 month, and his BMI was always $>2$ SDS for his age (5.43-13.07 SDS). BMI, body mass index; SDS, standard deviation score. 
We attempted to find an underlying genetic defect to account for his early-onset severe obesity and associated elevated liver enzymes and developmental delays. Targeted gene panel sequencing was performed to detect the presence of genes potentially associated with early-onset severe obesity, hepatic disorders, and developmental delay, including associated diseases such as leptin receptor deficiency, POMC deficiency, and Alström syndrome. The average read depth for each candidate gene is shown in Table 1. Informed consent was obtained from both parents. We obtained $3 \mathrm{~mL}$ of blood from the patient and both parents. Genomic DNA was extracted from the peripheral blood, and a cDNA library was prepared using the Celemics G-Mendeliome DES Panel (Celemics Inc., Seoul, Korea), which enriched a 12-Mb region spanning 70,418 target exons from a total of 5,447 clinically relevant genes. Massively parallel sequencing was performed on the Illumina
NextSeq platform. Sequence reads were mapped to a UCSC hg19 standard base for conducting a comparative analysis. The average depth of the panel was $133.17 \mathrm{X}$, and the percentage of bases above 10X of $A T P 7 B$ was $100.0 \%$.

We found compound heterozygous mutations for c.2513del (p.Lys838SerfsTer ${ }^{\star} 35$ ) and c.2333G $>\mathrm{T}$ (p.Arg778Leu) in ATP7B on chromosome 13q14.3. Sanger sequencing confirmed the presence of these variants, and one of the same heterozygous variants was found in his father and mother, respectively (Fig. 2). These variants have been previously associated with WD. Serum ceruloplasmin declined to $4.09 \mathrm{mg} / \mathrm{dL}$ (normal range, 20-46 $\mathrm{mg} / \mathrm{dL}$ ). Due to a lack of patient cooperation, a 24 -hour urine specimen could not be collected and the Kayser-Fleischer ring in the cornea could not be examined. We started trientine (750 $\mathrm{mg} /$ day) to treat $\mathrm{WD}$, and after 3 months of treatment, his liver enzyme began to decline (Fig. 3). Diet and exercise interventions
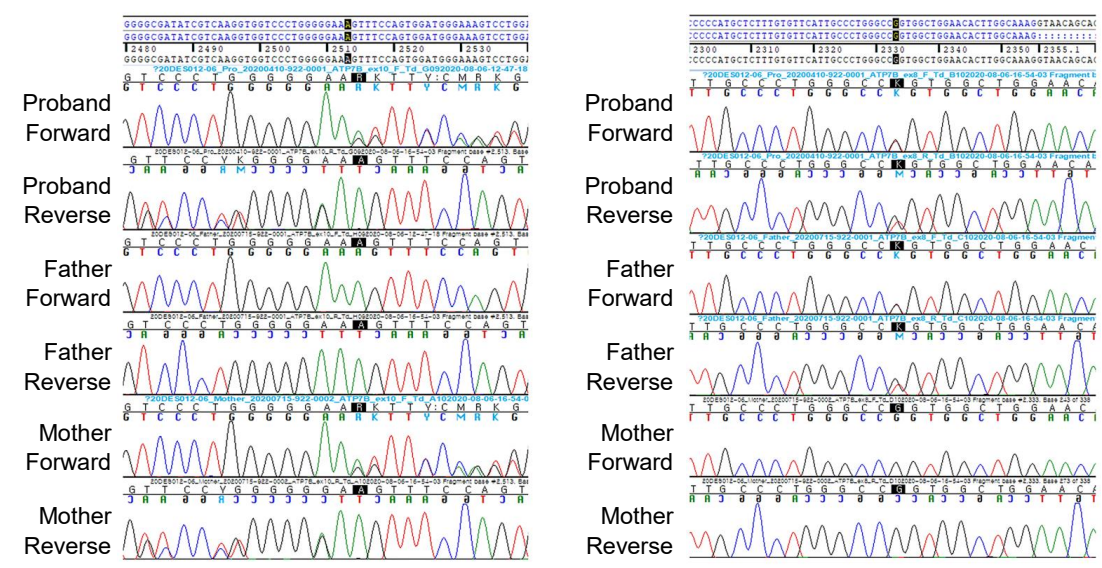

Fig. 2. Sanger sequencing confirmation of the compound heterozygous mutations for c.2513del (p.Lys838SerfSTer*35) and c.2333G>T (p.Arg778Leu) in the ATP7B gene on chromosome 13q14.3. One heterozygous variant was found in each of the patient's father and mother, respectively.

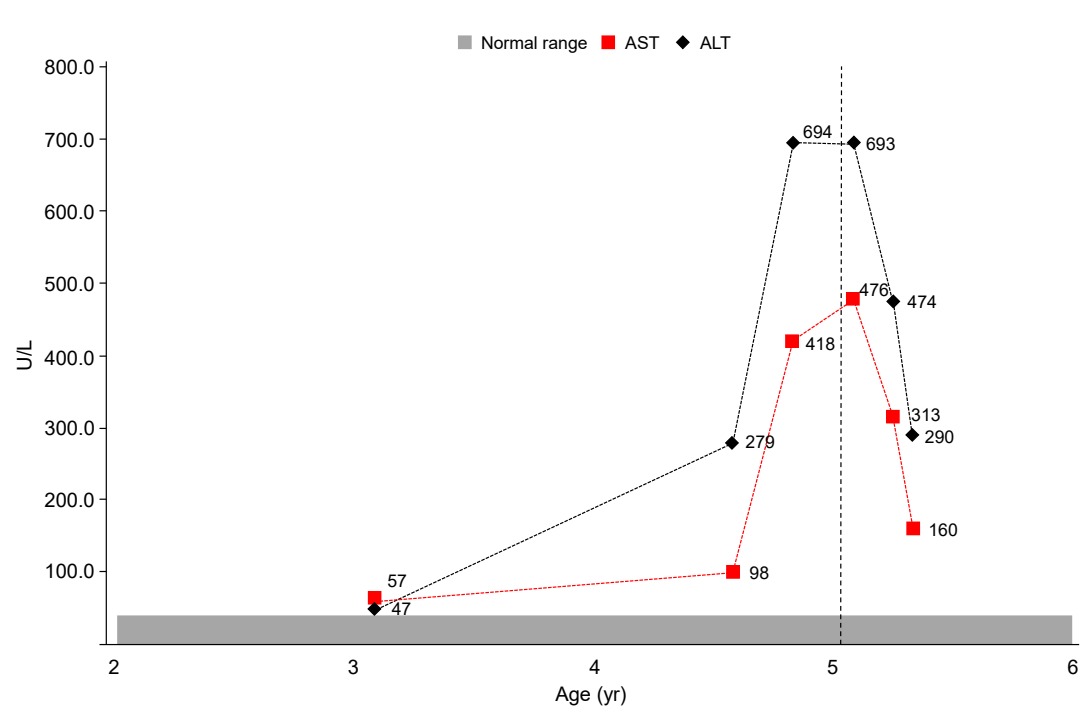

Fig. 3. Liver enzyme transition in the patient. After treatment began (at age 5 years 1 month, indicated by the dotted line), enzyme levels decreased. AST, aspartate aminotransferase; ALT, alanine aminotransferase. 


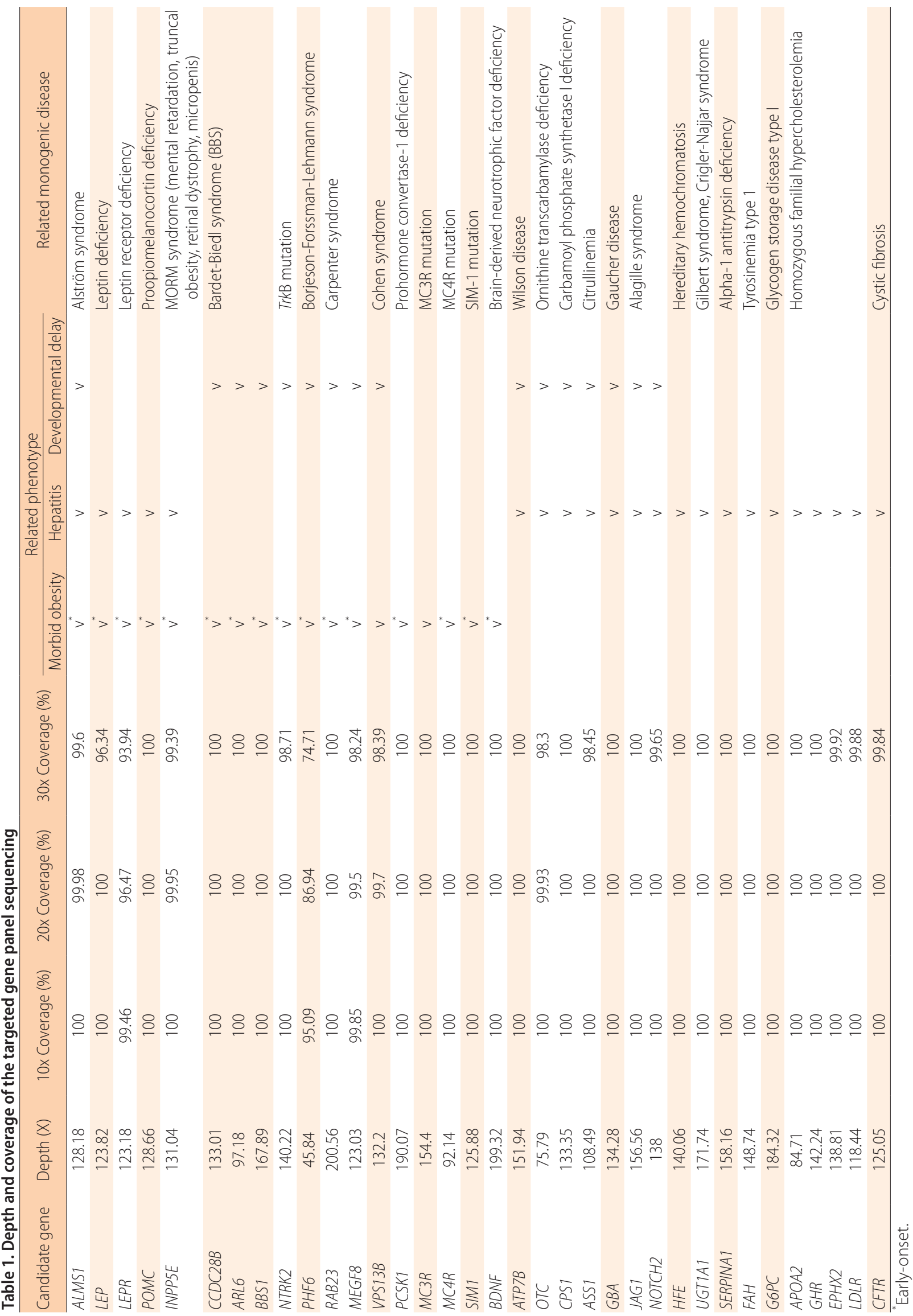


for severe obesity were initiated.

\section{Discussion}

In this study, we report a male aged 4 years 7 months with obese WD accompanied by NAFLD. We diagnosed him with WD incidentally based on targeted gene panel sequencing to screen for genes related to pathologic obesity. Trientine was effective in improving his liver function.

NAFLD is associated with obesity. Numerous studies have indicated that NAFLD accounts for the hepatic component of metabolic syndrome characterized by obesity, insulinresistance and type 2 diabetes, hypertriglyceridemia, and arterial hypertension. Although NAFLD was reported to be the most common liver pathology in WD patients, there are limited data on copper accumulation and NAFLD pathogenesis. ${ }^{3)}$ WD was first overlooked in our patient because of his morbid obesity and NAFLD. However, the most frequently observed hepatic histological lesion in WD is hepatic macro-steatosis and glycogenated nuclei, which are also features of NAFLD. ${ }^{2)}$ In a recently published study, hepatic steatosis in WD was not induced by metabolic comorbidities but by copper accumulation in liver tissue. ${ }^{3)}$ However, metabolic alterations could be cofactors in pathogenic steatosis in WD. Thus, NAFLD presence should not exclude a WD diagnosis.

Our patient was severely obese in early childhood. He typically enjoyed high-calorie foods, and his delayed motor development from cerebral palsy resulted in weight gain due to a lack of physical activity. A previous animal model study ${ }^{4)}$ suggested that WD may not be related to obesity. Atp7b-/- mice were more glucose tolerant and insulin sensitive and showed decreased hepatic steatosis, while chow-fed Atp $7 \mathrm{~b}-/-$ mice exhibited modest reduction in adiposity and body weight by 14 weeks of age compared with wild type mice. This suggests that obesity is less likely to develop in WD. Tribl et al. ${ }^{5)}$ also found that BMI was significantly lower in the WD group than in the control group in their case control study of sleep disorders in
WD.

A few WD cases associated with other endocrinologic problems have been reported (Table 2). Growth and puberty may be affected by chronic systemic illness. A few cases of infertility, anovulation, amenorrhea, and short stature have been reported in patients with WD. Hypogonadotropic hypogonadism secondary to chronic liver disease is the most common cause of gonadal dysfunction in patients with WD. ${ }^{6,7)}$ One case showed growth hormone deficiency. ${ }^{8}$ Kaushansky et al. .) $^{9}$ reported disturbed ovarian function in one WD family, as evidenced by low estradiol, high total testosterone, normal free testosterone, and elevated androstenedione. Another rare endocrinological disturbance is hypoparathyroidism, possibly due to copper accumulation in the parathyroid glands. ${ }^{10)}$ Krysiak et al. ${ }^{11)}$ reported 1 case of insulinoma and hyperprolactinoma in a female patient with WD, in which galactorrhea was the initial symptom 5 years before diagnosis. Golding et al. ${ }^{12}$ and Canelas et al. ${ }^{13)}$ reported radiographic evidence of osteoporosis in up to $88 \%$ of people with WD.

This patient's neurological manifestations were developmental delay and seizure, both of which are not frequently reported in patients with WD. Periventricular leukomalacia is also not typically observed in WD. However, several studies have shown white matter lesions in patients with WD, suggesting association with copper accumulation. Assessment for improvements in developmental delay after trientine treatment might lead to a better understanding of its relevance in WD.

WD is controllable with appropriate copper chelating agents. In most patients with WD, early diagnosis and treatment generally result in symptom resolution and improvements in quality of life, growth problems, gonadal dysfunction, or other endocrinologic problems. Serum ceruloplasmin has been considered a simple method for testing WD. However, up to 20\% of patients show normal or borderline ceruloplasmin level. ${ }^{14)}$ The 24-hour urinary copper excretion may be another useful tool for diagnosing WD and for monitoring treatment. However, ceruloplasmin and urinary copper levels are affected by acute infection, hepatic injury, renal failure, and certain medications

Table 2. Reported endocrine cases in Wilson disease

\begin{tabular}{|c|c|c|c|c|c|c|}
\hline No. & Endocrinologic problems & Age/sex & Country & Characteristic clinical findings & References & $\begin{array}{l}\text { Publication } \\
\text { year }\end{array}$ \\
\hline 1 & Hypopituitarism & $40 / F$ & South Korea & $\begin{array}{l}\text { Secondary hypothyroidism, adrenal insuffi- } \\
\text { ciency }\end{array}$ & Lee et al. ${ }^{16)}$ & 2016 \\
\hline 2 & Insulinoma, hyperprolactinemia & $24 / F$ & Poland & $\begin{array}{l}\text { Galactorrhea, menstrual abnormalities, hypo- } \\
\text { glycemia, partial response to bromocriptine }\end{array}$ & Krysiak et al. ${ }^{11)}$ & 2012 \\
\hline 3 & GHD & $10 / \mathrm{M}$ & Germany & $\begin{array}{l}\text { After starting chelation treatment, IGF-1 } \\
\text { normalized; not from GH treatment. }\end{array}$ & Koch et al. ${ }^{8)}$ & 1995 \\
\hline 4 & Amenorrhea oligomenorrhea & $17 / F, 32 / F$ & Korea & Low estradiol, high total testosterone & Lee et al. ${ }^{17)}$ & 1990 \\
\hline 5 & $\begin{array}{l}\text { Severe oligomenorrhea or } \\
\text { amenorrhea }\end{array}$ & $\begin{array}{l}15 / F, 16 / F \\
9 / F, 10 / F\end{array}$ & Israel & Low estradiol, high total testosterone & Kaushansky et al.9) & 1987 \\
\hline 6 & Hypoparathyroidism & $11 / F$ & USA & Fanconi syndrome & Carpenter et al..$^{10)}$ & 1983 \\
\hline 7 & Unexplained miscarriage & NA & UK & NA & $\mathrm{Klee}^{18)}$ & 1979 \\
\hline 8 & $\begin{array}{l}\text { Menstrual irregularity, delayed } \\
\text { puberty, gynecomastia }\end{array}$ & NA & USA & NA & Scheinberg and Sternlieb ${ }^{19)}$ & 1965 \\
\hline
\end{tabular}

GHD, growth hormone deficiency; IGF-1, insulin-like growth. factor-1; GH, growth hormone; NA, not applicable. 
such as steroids and estrogens. Moreover, Cauza et al. ${ }^{15)}$ found that the positive predictive value of low serum ceruloplasmin level was only 5.9\%. These diagnostic tests are usually performed on those with newly onset hepatic abnormalities because serum AST generally increases in WD, except at very early disease stages. Only about $40 \%$ of pediatric patients present with hepatic symptoms. ${ }^{7)}$ In this respect, WD diagnosis could be challenging, especially in young patients or during the early disease phase.

Our patient was diagnosed with WD using a commercialized targeted gene panel that contained the WD-related gene. Although genetic testing for NAFLD in general cases is not yet recommended, the genetic etiology of NAFLD-related metabolic syndrome has been extensively studied. In WD, for nonspecific early symptoms such as NAFLD, generalizing next-generation sequencing in parallel with copper analysis is a promising step for early diagnosis. As genetic testing technology has been widely used, screening for asymptomatic families is gradually increasing, and the diagnosis age for WD has decreased to approximately $2-3$ years. Direct sequencing is a highly sensitive and widely used confirmation test method, and simultaneous biochemical testing improves its diagnostic accuracy. However, it is not widely available due to its cost.

In conclusion, increased liver enzymes are relatively common in obese children. A WD diagnosis should not be overlooked even in NAFLD cases that appear with obesity because WD can present as any liver disease.

\section{Notes}

Conflicts of interest: No potential conflict of interest relevant to this article was reported.

Funding: This study received no specific grant from any funding agency in the public, commercial, or not-for-profit sectors.

Author contribution: Conceptualization: MI, SYC, DJ; Data curation: MI, SL, SYC; Formal analysis: MI; Methodology: MI, SYC; Project administration: MI, SYC; Visualization: MI, SYC; Writing - original draft: MI; Writing - review \& editing: MI, AS, JK, MK, SL, MJK, SYC

\section{ORCID}

Minji Im: 0000-0002-8114-844X

Ari Song: 0000-0003-3689-5312

Jiyeon Kim: 0000-0002-6612-1732

Min-Sun Kim: 0000-0002-7799-3229

Sae-Mi Lee: 0000-0002-0462-6700

Mi Jin Kim: 0000-0002-4505-4083

Sung Yoon Cho: 0000-0003-2913-059X

Dong-Kyu Jin: 0000-0003-4162-2706

\section{References}

1. Scheinberg IS, Sternlieb I. Wilson's disease (a volume in the major problems in internal medicine series). Philadelphia (PA): W. B. Saunders; 1984.p. 192.
2. Ludwig J, Moyer TP, Rakela J. The liver biopsy diagnosis of Wilson's disease. Methods in pathology. Am J Clin Pathol 1994;102:443-6.

3. Liggi M, Murgia D, Civolani A, Demelia E, Sorbello O, Demelia L. The relationship between copper and steatosis in Wilson's disease. Clin Res Hepatol Gastroenterol 2013;37:36-40.

4. Wooton-Kee CR, Robertson M, Zhou Y, Dong B, Sun Z, Kim KH, et al. Metabolic dysregulation in the Atp7b (-/) Wilson's disease mouse model. Proc Natl Acad Sci U S A 2020;117:2076-83.

5. Tribl GG, Trindade MC, Bittencourt T, Lorenzi-Filho G, Cardoso Alves R, Ciampi de Andrade D, et al. Wilson's disease with and without rapid eye movement sleep behavior disorder compared to healthy matched controls. Sleep Med 2016;17:179-85.

6. Sinha S, Taly AB, Prashanth LK, Arunodaya GR, Swamy HS. Successful pregnancies and abortions in symptomatic and asymptomatic Wilson's disease. J Neurol Sci 2004;217:3740.

7. Ala A, Walker AP, Ashkan K, Dooley JS, Schilsky ML. Wilson's disease. Lancet 2007;369:397-408.

8. Koch A, Dörr HG, Gerlin S, Behrens R, Böhles HJ. Effect of growth hormone on IGF-I levels in a patient with growth hormone deficiency and Wilson disease. Horm Res 1995;44:40-4.

9. Kaushansky A, Frydman M, Kaufman H, Homburg R. Endocrine studies of the ovulatory disturbances in Wilson's disease (hepatolenticular degeneration). Fertil Steril 1987;47:270-3

10. Carpenter TO, Carnes DL, Anast CS. Hypoparathyroidism in Wilson's disease. N Engl J Med 1983;309:873-7.

11. Krysiak R, Handzlik-Orlik G, Okopien B. Endocrine symptoms as the initial manifestation of Wilson's disease. Yale J Biol Med 2012;85:249-54.

12. Golding DN, Walshe JM. Arthropathy of Wilson's disease. Study of clinical and radiological features in 32 patients. Ann Rheum Dis 1977;36:99-111.

13. Canelas HM, Carvalho N, Scaff M, Vitule A, Barbosa ER, Azevedo EM. Osteoarthropathy of hepatolenticular degeneration. Acta Neurol Scand 1978;57:481-7.

14. Nicastro E, Loudianos G, Zancan L, D’Antiga L, Maggiore G, Marcellini M, et al. Genotype-phenotype correlation in Italian children with Wilson's disease. J Hepatol 2009;50: 555-61.

15. Cauza E, Maier-Dobersberger T, Polli C, Kaserer K, Kramer L, Ferenci P. Screening for Wilson's disease in patients with liver diseases by serum ceruloplasmin. J Hepatol 1997;27:358-62.

16. Lee HW, Kang JD, Yeo CW, Yoon SW, Lee KJ, Choi MK. Hypopituitarism presenting as adrenal insufficiency and hypothyroidism in a patient with wilson's disease: a case report. J Korean Med Sci 2016;31:1345-8. 
17. Lee YK, Kim SH, Hah JS, Park CS. Endocrine study on menstrual irregularities in Wilson's Disease. J Korean Neurol Assoc 1990;8:312-7.

18. Klee JG. Undiagnosed Wilson's disease as cause of unexplained miscarriage. Lancet 1979;2:423.

19. Scheinberg IH, Sternlieb I. Wilson's disease. Annu Rev Med 1965;16:119-34. 